\title{
Coupling high-throughput experiments and regression algorithms to optimize PGM-free ORR electrocatalyst synthesis
}

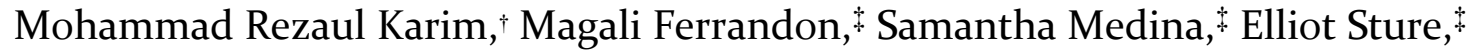 \\ Nancy Kariuki, $₫$ Deborah J. Myers, ${ }^{*}$, Edward F. Holby, ${ }^{\nabla}$ Piotr Zelenay, ${ }^{\S}$ Towfiq Ahmed ${ }^{*} \uparrow$ \\ †Los Alamos National Laboratory, Theoretical Division, Los Alamos, NM 87545, USA \\ *Argonne National Laboratory, Chemical Sciences and Engineering Division, Lemont, IL, 60439, USA \\ ${ }^{\nabla}$ Los Alamos National Laboratory, Sigma Division, Los Alamos, NM 87545, USA \\ $\S$ Los Alamos National laboratory, Materials Physics and Applications Division, Los Alamos, NM 87545, USA
}

* Corresponding author(s) to whom correspondence should be addressed:

Towfiq Ahmed: atowfiq@lanl.gov; Deborah J. Myers: dmyers@anl.gov

\section{Experimental}

Synthesis

The catalyst-ionomer inks were prepared by mixing 2 mg of the catalyst powder with $2.2 \mathrm{~mL}$ Isopropanol (99.5\% ACS Reagent, Sigma-Aldrich) and $10 \mu \mathrm{L}$ of $5 \mathrm{wt} \%$ Nafion $^{\mathrm{TM}}$ in lower aliphatic alcohols and water $\left(\mathrm{DE}_{520}\right.$, EW10oo, $5 \mathrm{wt} \%$ and $45 \mathrm{wt} \%$ water, $0.924 \mathrm{~g} / \mathrm{mL}$, SigmaAldrich). The inks were mixed in a $5 \mathrm{~mL}$ glass vial by sonicating in an ice bath until visually the ink appeared to be free of large catalyst agglomerates (required at least two hours of sonication). Immediately following mixing, the inks were deposited on either the glassy carbon electrode of a rotating disk electrode (RDE, $5 \mathrm{~mm}$ O.D., $0.196 \mathrm{~cm}^{2}$, embedded in a PTFE cylinder, Pine Instruments) or onto a $3 \mathrm{~mm} \times 1 \mathrm{~mm}$ rectangular glassy carbon electrode in a custom-made electrode assembly of a custom-designed and constructed multi-channel flow double electrode cell (m-CFDE) that allowed the highthroughput analysis of the ORR activity of four catalyst samples in rapid succession. ${ }^{1}$ The ink deposition procedure for the RDE measurements is described in detail in Kocha et al..2 The volume of ink deposited was chosen to obtain a catalyst loading on the glassy carbon electrode of $0.6 \mathrm{mg} \mathrm{cm}^{-2}$. Following air-drying of the ink, the electrodes were immersed in Ar-saturated $0.5 \mathrm{M}$ $\mathrm{H}_{2} \mathrm{SO}_{4}$ (98.3\% sulfuric acid sourced from Veritas ${ }^{\circledR}$ Doubly Distilled, GFS chemicals) in either the m-CFDE or a standard three-electrode RDE cell. The counter electrode was a carbon rod and the reference electrode was a mercury-mercurous sulfate electrode (Koslow Scientific Company, Goo93; filling solution, saturated potassium sulfate) located in a salt bridge (Gamry, 930-00045; filling solution, $0.5 \mathrm{M}$ sulfuric acid) that was inserted directly into the main cell compartment. The reference electrode was calibrated versus a platinum wire immersed in a hydrogen-saturated $0.5 \quad \mathrm{M} \quad \mathrm{H}_{2} \mathrm{SO}_{4}$ solution (i.e., a reversible hydrogen electrode (RHE)). All potentials are reported versus RHE. Cyclic voltammograms (CVs) of the catalyst-ionomer deposits were acquired in Ar-saturated $0.5 \mathrm{M} \mathrm{H}_{2} \mathrm{SO}_{4}$ over the o.0 and 1.0 $\mathrm{V}$ (vs RHE) potential range at a scan rate of $5 \mathrm{mV} \mathrm{s}^{-1}$ and with $\mathrm{iR}$ correction using a $\mathrm{CHI} 760$ potentiostat ( $\mathrm{CH}$ Instruments, Inc.). $\mathrm{CVs}$ were then acquired over the same potential range and at the same scan rate in oxygen-saturated $0.5 \mathrm{M} \mathrm{H}_{2} \mathrm{SO}_{4}$ electrolyte at an RDE rotation rate of $900 \mathrm{rpm}$ and, in the $\mathrm{m}$-CFDE, an electrolyte flow rate that provided the same mass transport-limited ORR current density as observed in the RDE traces. The $\mathrm{CV}$ taken in Ar-saturated electrolyte was subtracted from that taken in oxygensaturated electrolyte to obtain the background-corrected CV. A process flow diagram is shown in Figure S1. The ORR mass activity at $0.75 \mathrm{~V}$ was extracted from the background-corrected CV using the following well-known equation for mass-transport correction of RDE data

$$
\text { ORR MA }=\frac{\left(\frac{\mathrm{I}_{\lim } \mathrm{I}_{0.75}}{\mathrm{I}_{\lim }-\mathrm{I}_{0.75}}\right)}{\left(0.196 \mathrm{~cm}^{2} \times 0.60 \mathrm{mg} / \mathrm{cm}^{2}\right)}
$$

There was good agreement between the m-CFDE and RDE-derived ORR MA values and the data from both types of experiments were used as input to the machine learning models as shown in Figure $\mathbf{S}_{2}$. Figure $\mathbf{S}_{\mathbf{3}}$ shows 
polarization curves from which the mass activity are derived.

\section{Machine learning regression}

A reliable quantitative way to understand the machine learning based prediction is to perform a sensitivity analysis of the surrogate models. For each of the ML based surrogate models investigated in this work, we performed sensitivity analysis to examine how each feature affects the model. Among all the features, pyrolysis temperature was found to have a major impact on the target function (ORR mass activity). We trained five different machine learning models on the training data set. These are: (i) Gradient Boosting Regression with least absolute deviation loss and 500 regression trees of depth 5 and learning rate 0.2 , (ii) Random Forest Regression with 100 forest trees of depth 5 and trees build with bootstrap samples, (iii) Polynomial Regression with default features of degree 2, (iv) Support Vector Regression with radial basis kernel (rbf) function and (v) Neural Network Regression with 100 number of neurons in the hidden layer and lbfgs solver which is an optimizer in the family of quasi-Newton methods. Then, we developed different surrogate models using following multiple steps:

First, we randomly sampled $80 \%$ of the highthroughput synthesis data as the "training data" set where the machine learning based surrogate models are trained. The rest of the $20 \%$ data is called "test data" where models predictions are tested. We used five different machine learning algorithms to train our surrogate models on a randomly selected training data set. Prediction uncertainty is then quantified by comparing and calculating root-mean-square-error (RMSE) between the model-predicted values with true values (experimental ORR) on the $20 \%$ test data set. However, surrogate models trained on a single shot randomly selected 'training data' is not guaranteed to perform optimally with high accuracy (low RMSE) prediction. In the second step, we address this issue by implementing a novel iterative approach where training data sets are randomly selected multiple times. We notice the error uncertainty (RMSE) depends on the total number of random sampling (or iterations). For larger number of iterations, the RMSE values tend to converge (Figure 1 in main text). However, the RMSE value from different ML based surrogate models tend to converge at different rates (i.e. different number of total iterations) as shown in

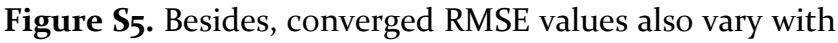
the ML algorithm used to generate the surrogate models. Calculated RMSE values among various surrogate models are compared in Figure S6. Out of the five ML based surrogate models we benchmarked in this work, the gradient-boosting and support vector regression based models clearly outperformed the others with a mean absolute percent error (MAPE) less than $4.5 \%$ and $7 \%$ respectively (Table $\mathbf{1}$ in main text).

To investigate the accuracy of the constructed surrogate models, we plot the experimentally-determined ORR mass activity as a function of predicted ORR mass activity in main text Figure 2. The blue dashed line represents an ideal fit, and the red dotted circles are a measure of deviation between true (experimental) and predicted values of ORR for the RMSE optimized ML based surrogate models 


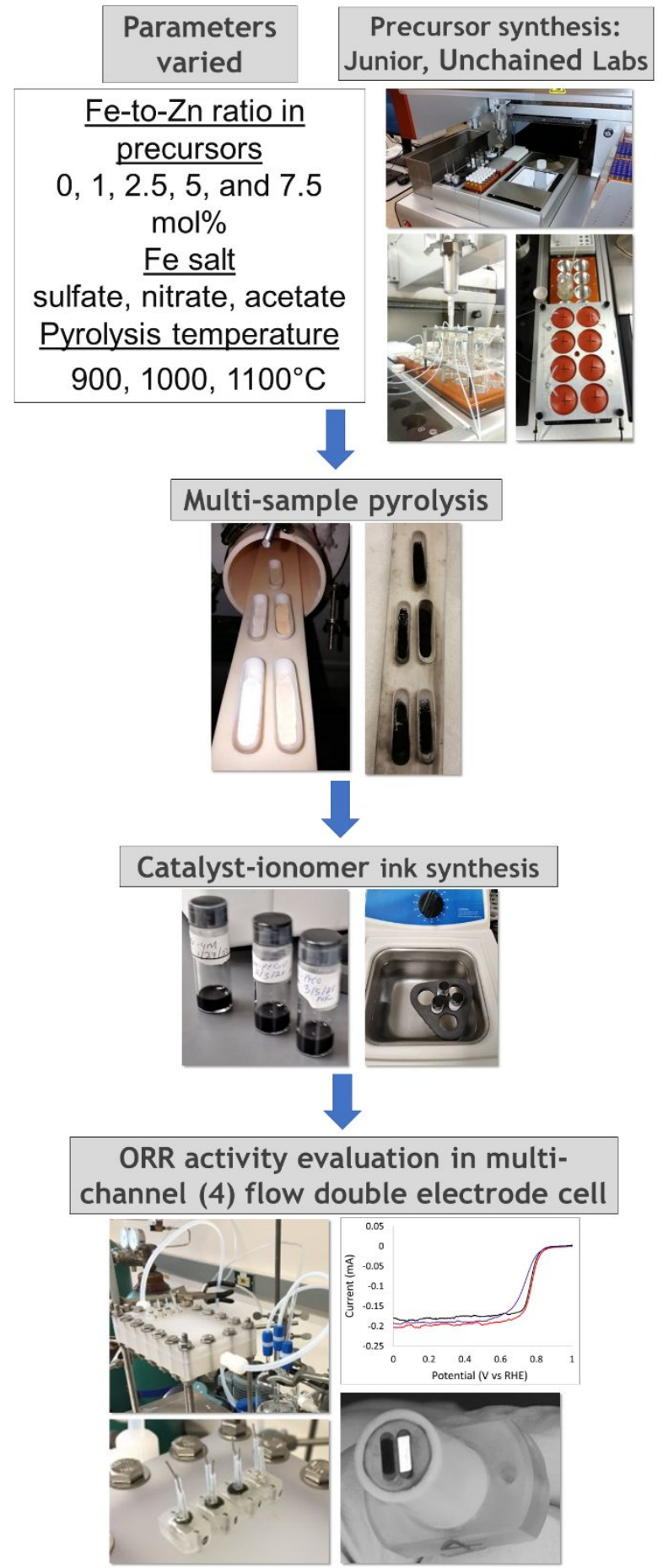

Figure S1. A schematic of the process and experimental set-ups. 


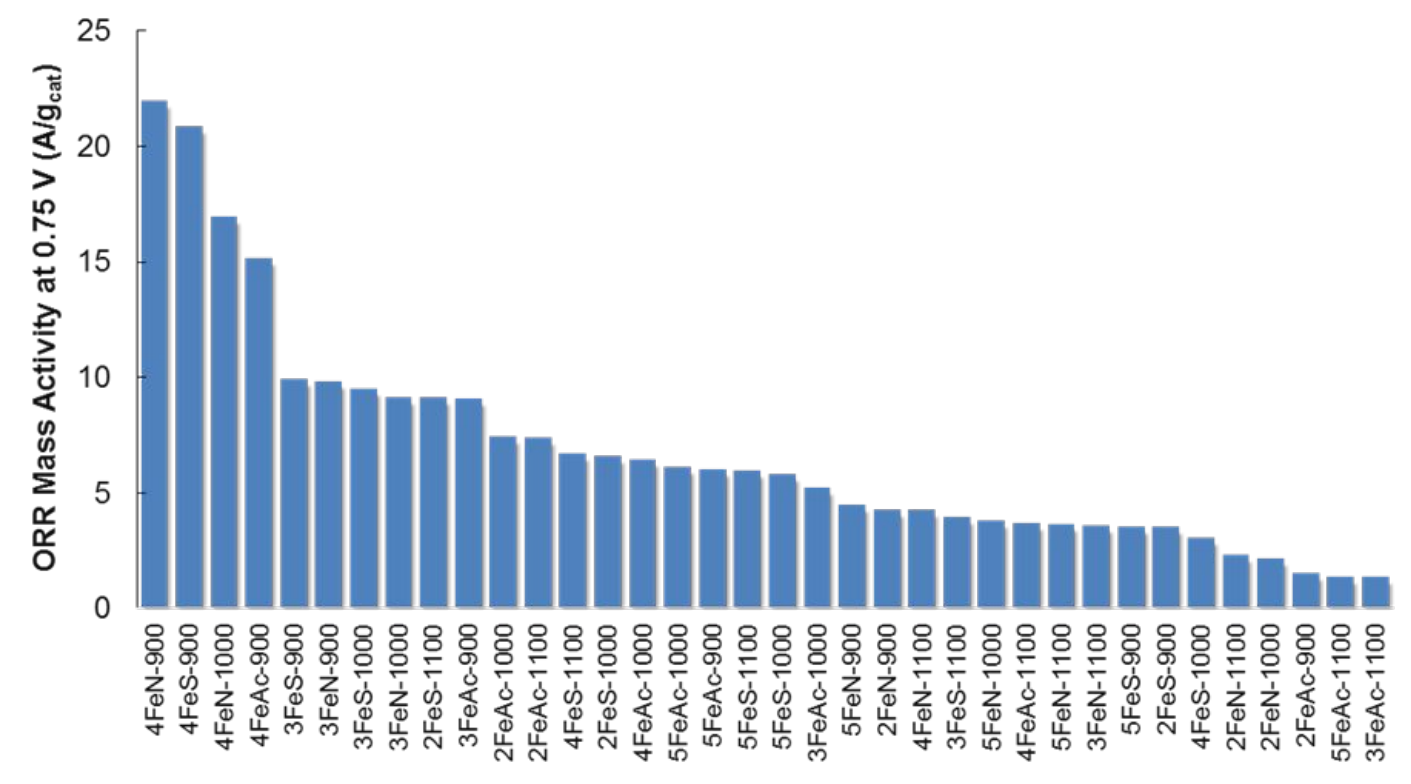

Figure S2. Graphic representation of experimental data: ORR mass activity vs. three input control parameters (Fe precursor metal fraction, FeN precursor, and pyrolysis temperature)

Table S1. Experimental ORR mass activity for 36 catalysts. Here, $(\mathrm{Fe} / \mathrm{Fe}+\mathrm{Zn}) \times 100(\mathrm{Fe} \%)$ is the atomic percent of Fe in the sum of $\mathrm{Fe}+\mathrm{Zn}$, FePre (atoms/mol) defines Fe precursor as a number of atoms in the precursor molecule (where Fe acetate $=15$ atoms per molecule, Fe sulfate $=27$ atoms per hydrated molecule, and Fe nitrate $=40$ atoms per hydrated molecule) and $\mathrm{T}$ is the pyrolysis temperature.

\begin{tabular}{|c|c|c|c|}
\hline $\mathrm{T}$ & $(\mathrm{Fe} / \mathrm{Fe}+\mathrm{Zn}) \times \mathbf{1 0 0}(\mathrm{Fe} \%)$ & FePre (atoms/molecule) & $\begin{array}{l}\text { ORR at } 0.75 \mathrm{~V} \\
\text { (A/gcatalyst) }\end{array}$ \\
\hline \multirow{11}{*}{900} & \multirow{3}{*}{2.5} & 40 & 9.91 \\
\hline & & 27 & 9.85 \\
\hline & & 15 & 9.08 \\
\hline & \multirow{3}{*}{5} & 40 & 21.98 \\
\hline & & 27 & 20.89 \\
\hline & & 15 & 15.11 \\
\hline & \multirow{3}{*}{$7 \cdot 5$} & 40 & 4.51 \\
\hline & & 27 & 3.54 \\
\hline & & 15 & 6.04 \\
\hline & \multirow[b]{2}{*}{1} & 40 & 4.26 \\
\hline & & 27 & 3.53 \\
\hline
\end{tabular}




\begin{tabular}{|c|c|c|c|}
\hline & & 15 & 1.52 \\
\hline \multirow{12}{*}{1000} & \multirow{3}{*}{2.5} & 40 & 9.16 \\
\hline & & 27 & 9.51 \\
\hline & & 15 & 5.21 \\
\hline & \multirow{3}{*}{5} & 40 & 16.94 \\
\hline & & 27 & 3.07 \\
\hline & & 15 & 6.46 \\
\hline & \multirow{3}{*}{7.5} & 40 & 3.81 \\
\hline & & 27 & 5.81 \\
\hline & & 15 & 6.11 \\
\hline & \multirow{3}{*}{1} & 40 & 2.18 \\
\hline & & 27 & 6.58 \\
\hline & & 15 & 7.43 \\
\hline \multirow{11}{*}{1100} & \multirow{3}{*}{2.5} & 40 & 3.59 \\
\hline & & 27 & 3.99 \\
\hline & & 15 & 1.37 \\
\hline & \multirow{3}{*}{5} & 40 & 4.25 \\
\hline & & 27 & 6.71 \\
\hline & & 15 & 3.71 \\
\hline & \multirow{3}{*}{$7 \cdot 5$} & 40 & 3.62 \\
\hline & & 27 & 5.99 \\
\hline & & 15 & 1.38 \\
\hline & \multirow[b]{2}{*}{1} & 40 & 2.30 \\
\hline & & 27 & 9.15 \\
\hline
\end{tabular}




\begin{tabular}{|l|l|l|l|}
\hline & & 15 & $7 \cdot 4$ \\
\hline
\end{tabular}
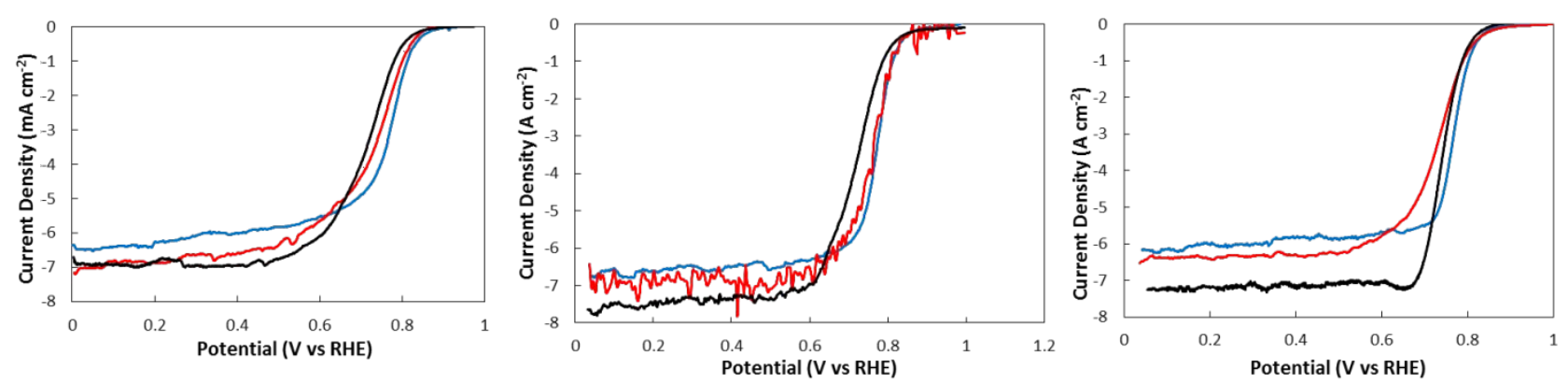

Figure S3. ORR voltammetry of Fe-N-C catalysts acquired using the mCFDE. Conditions: $0.6 \mathrm{mgcm}^{-2} \mathrm{catalyst}_{\text {in }}$ oxygensaturated $0.5 \mathrm{H}_{2} \mathrm{SO}_{4}$ electrolyte, room temperature, $5 \mathrm{mVs}^{-1}$, iR-corrected. Ctalysts: 5 at\% Fe; Fe-acetate (left), Fe nitrate (middle), and Fe sulfate (right) precursors, pyrolyzed at $900{ }^{\circ} \mathrm{C}$ (blue), $1000^{\circ} \mathrm{C}$ (red), and $1100{ }^{\circ} \mathrm{C}$ (black).

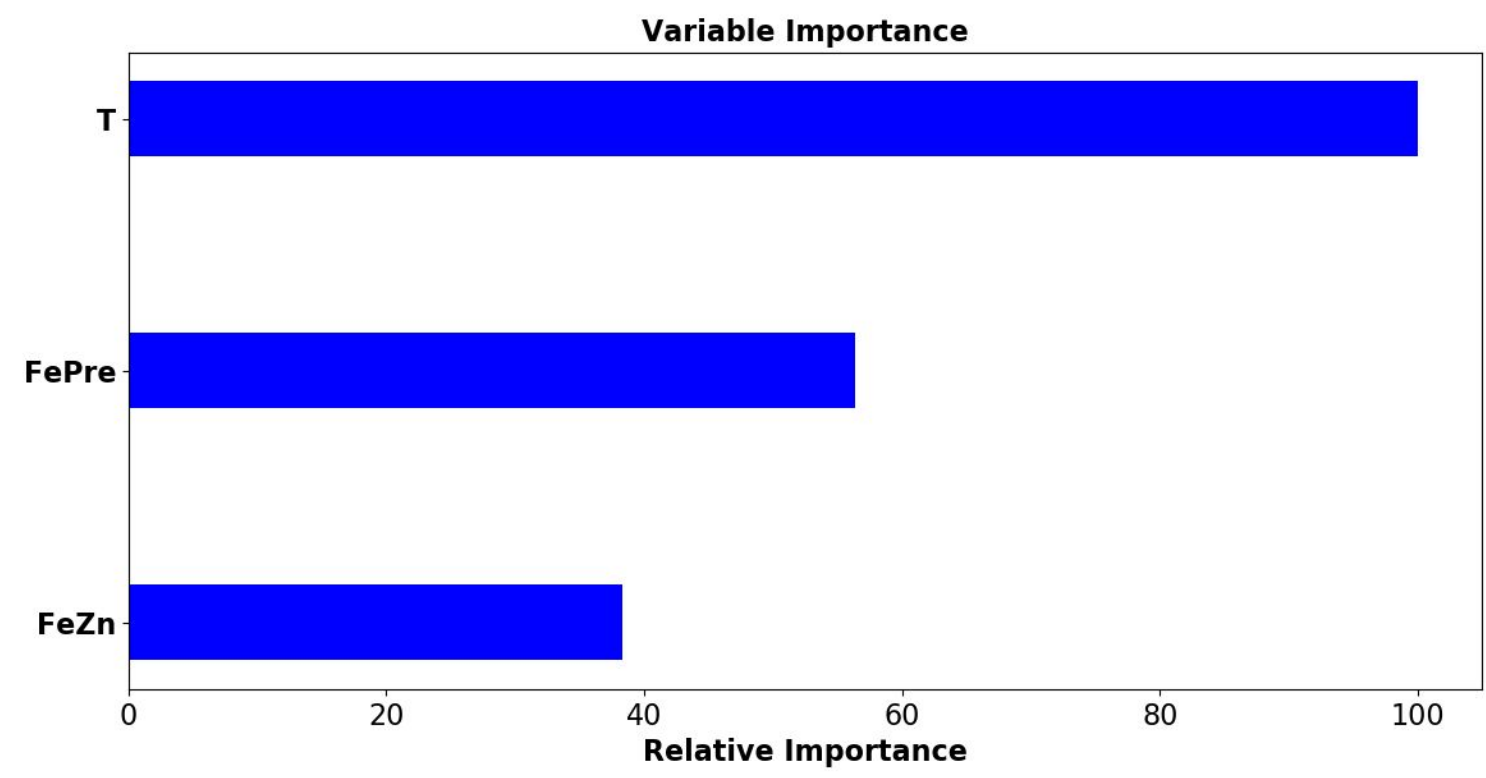

Figure S4. Relative importance of synthesis parameters. 


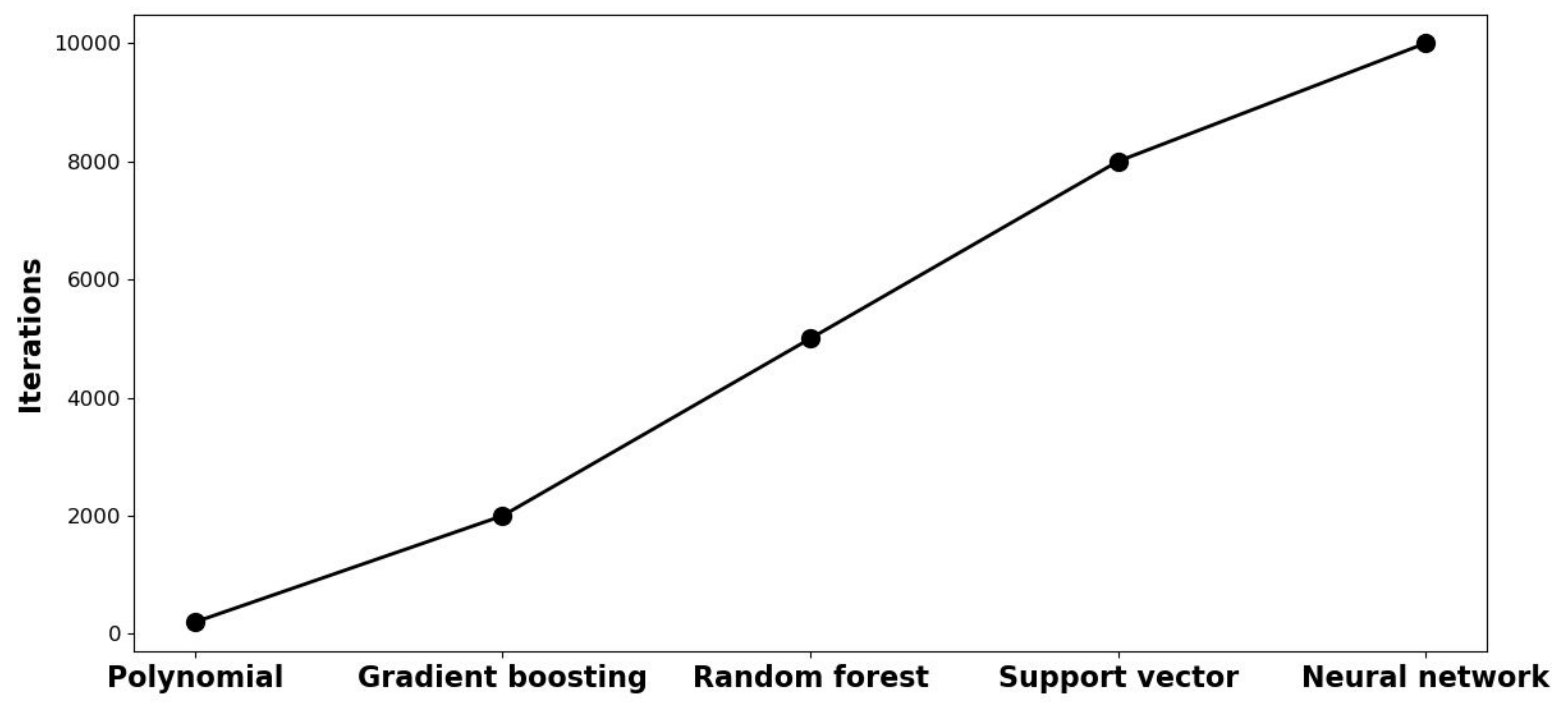

Figure S5: Total number of iteration needed to achieve RMSE convergence: a comparison between different ML algorithms.

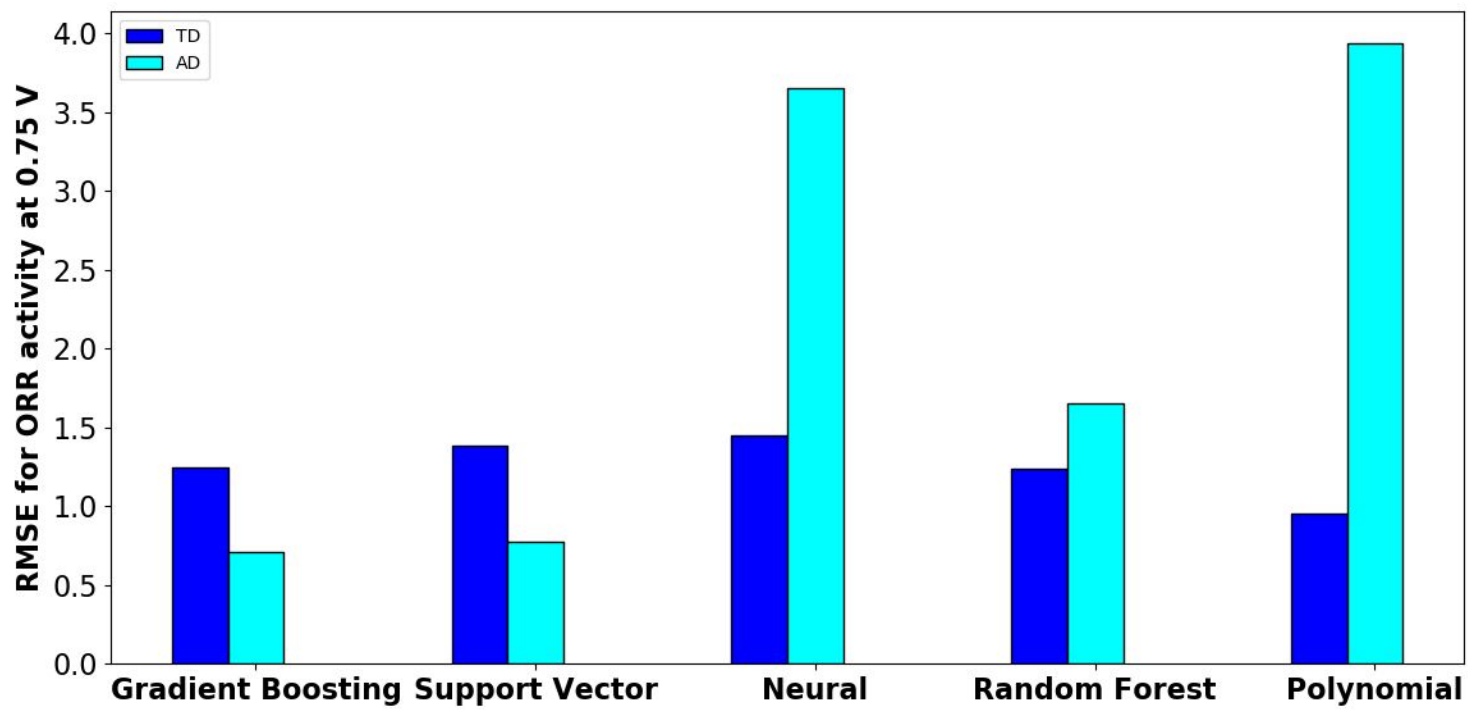

Figure S6. RMSE value as a function of machine learning based models; TD=test dataset and $\mathrm{AD}=36$-sample dataset.

\section{References}

(1) Zelenay, P. Electrocat (Electrocatalysis Consortium). Electrocat (Electrocatalysis Consortium); https://www.hydrogen.energy.gov/pdfs/review17/fc16o_zelena \ـ2017_0.pdf

2017, 40 .
(2) Kocha, S. S.; Shinozaki, K.; Zack, J. W.; Myers, D. J.; Kariuki, N. N.; Nowicki, T.; Stamenkovic, V.; Kang, Y.; Li, D.; Papageorgopoulos, D. Best practices and testing protocols for benchmarking ORR activities of fuel cell electrocatalysts using rotating disk electrode. Electrocatalysis 2017, 8 (4), 366-374. 